\title{
TEMPERATURE AND MOVING BOUNDARY IN TWO-PHASE FREEZING DUE TO AN AXISYMMETRIC COLD SPOT*
}

\author{
BY \\ S. C. GUPTA \\ Indian Institute of Science, Bangalore
}

\begin{abstract}
Short time analytic solution of the problem of two-phase freezing due to an axisymmetric cold spot is presented. The melt could be superheated and it occupies an infinite region bounded internally by a cylinder of finite radius. Although the method of solution is valid for various other types of boundary conditions, the results in the paper are given for prescribed flux which could be time and space dependent. The method of solution is simple and straightforward and consists of assuming fictitious initial temperatures in some fictitious extensions of the region originally occupied by the melt. The spread of the solidification is much faster along the surface of the cylinder than along the interior of the cylinder and the spread along the surface always depends on material parameters. Several interesting results can be deduced as particular cases of the general results.
\end{abstract}

1. Introduction. A typical feature of the solidification problems (the melting problem is mathematically analogous to the solidification problem and it is sufficient to discuss here only solidification problems) is that, apart from the fixed boundary of the region originally occupied by the melt, there is also a boundary inside the region which is associated with the change of phase and is commonly known as the moving boundary. This moving boundary is unknown and is time dependent, and certain boundary conditions are to be satisfied on this moving boundary. Considerable information on the utility and analytical and numerical solutions of these moving boundary problems is contained in $[1-3]$.

According to the change of phase along the fixed boundary of the region originally occupied by the melt, Sikarskie and Boley [4] have classified the solidification problems into three classes. In class I problems, the solidification starts simultaneously at all points of the fixed boundary. In class II and III problems, the solidification initiates over a portion of the fixed boundary and at a point of the boundary, respectively. The problem considered in the present work is a class III problem. As compared to the problems of

\footnotetext{
* Received February 13, 1985.
} 
other classes, class I problems are easiest to tackle for the reason that, under suitable assumptions, many problems can be formulated as one-dimensional problems, but class II and III problems have to be at least two-dimensional. Further, in class I problems, a single boundary condition could be prescribed over the entire fixed boundary, whereas in other problems the boundary condition on the solidified portion of the fixed boundary will be different from the boundary condition on the portion of the boundary on which the solidification has not yet started. In one of the articles Boley [5] has stressed the need to study the multiphase problems of class II and III types for which, as far as we know, no analytical solution exists in the literature. However, for one-phase ablation problems (melting problems in which the melt is removed as soon as it is formed) some references exist [6-8].

The exact analytical solutions valid for total solidification time for multi-dimensional and multi-phase problems are rather unconceivable even for class I problems. For one-dimensional class I problems, some of the important analytical techniques employed are (i) similarity solutions such as the one given in [9], (ii) series solutions by Tao [10], (iii) perturbation solutions [11], and (iv) approximate methods [12]. Several other references can be found in the references listed above. None of the above-mentioned methods have been applied to class II and III problems and there does not seem to be any systematic way of extending these techniques to these classes of problems and so we shall not dwell on these methods. In the absence of analytical solutions valid for longer time, short time analytical solutions have also been developed [5, 13, 14] which are of considerable mathematical and practical interest. Class III problems may arise in many physical situations such as resistance spot welding, solidification of casting resulting from any unavoidable initial evenness in the mold, etc. In the laser welding process the interaction time is generally less than $0.1 \mathrm{~s}$ and the melt depth is generally less than $1 \mathrm{~mm}$ [15]. Short time solutions are ideally suited for direct applications or simulation of experimental runs in such processes.

Boley et al. [5] have contributed substantially in developing short time analytical solutions of several types of solidific/melting problems. Some of the papers of Boley et al. [6-8] are more relevant to the present work as they deal with the one-phase problems of melting due to hot spots and so we shall briefly describe the method of solution employed in these. In [16] Boley gave an embedding technique which consists of prescribing fictitious flux in the case of ablation problems and fictitious boundary flux (or fictitious boundary temperature) together with suitable fictitious initial temperature in the case of solidification problems. The solid and liquid regions are embedded in the region originally occupied by the melt. The temperature solution is written with the help of Duhamel's theorem [17] and the unknowns are determined by evaluating integrals and by comparing different powers of time variables and/or space variables on both sides of the equations. For one-phase class III melting problems in [6-8], Boley's embedding technique was suitably modified to account for the growth of the melting along the surface and toward the interior of the solid region. Some results on the uniqueness of these solutions are reported in [18].

The temperature solution in the present paper is written with the help of the source solution. Solid and liquid regions are first embedded in the region originally occupied by 
the melt, and then the original melt region is further extended fictitiously and fictitious initial temperatures are then prescribed for the extended fictitious regions. Unknown quantities are then determined by repeated differentiations of the equations with respect to the time variable and appropriate limits are taken. Both Boley's technique and the present technique are essentially integral-equation techniques.

2. Problem formulation. A superheated melt at time $t=0$ occupies the cylindrical mold region $R_{0} \leqslant r<\infty,|z|<\infty$ (axisymmetric problem in which $r$ and $z$ are cylindrical polar coordinates). This melt is cooled by prescribing a known flux $\hat{Q}_{\mathrm{L}}(z, t)$ on the surface $r=R_{0}$ of the cylinder. The temperature of the melt at $t=0$ is a known quantity $\hat{f}_{\mathrm{L}}(r, z)$ which is symmetric in $z$ and is such that

$$
\begin{gathered}
\hat{f}_{\mathrm{L}}(r, z) \begin{cases}=T_{m} ; & z=0, r=R_{0} \\
>T_{m} ; & z \neq 0, r \neq R_{0} .\end{cases} \\
\frac{\partial \hat{f}_{\mathrm{L}}}{\partial z}=0, \quad z=0 ; \quad \frac{\partial^{2} \hat{f}_{\mathrm{L}} \neq 0 \quad \text { at } r=R_{0}, z=0 .}{\partial z^{2}} .
\end{gathered}
$$

$T_{m}$ is the unique solidification temperature, $T_{m} \neq 0$. The solidification will start immediately at $z=0, r=R_{0}$, and with time it will grow along the surface $r=R_{0}$ of the cylinder and toward the interior of the cylinder. The equation of the solidification front may be written in the following mathematical form:

$$
r=s(z, t) ; \quad|z| \leqslant b(t) ;
$$

$|z|=b(t)$ is the equation of the spread of solidification along the surface $r=R_{0}$ of the cylinder, $s(z, t)$ and $b(t)$ are unknowns and are to be determined together with the temperatures in the liquid and solid regions by solving the following system of equations.

For liquid regions

$$
\begin{gathered}
2 \alpha^{2} \frac{\partial T_{\mathrm{L}}}{\partial V}=V\left(\frac{\partial^{2} T_{\mathrm{L}}}{\partial R^{2}}+\frac{1}{R} \frac{\partial T_{\mathrm{L}}}{\partial R}+\frac{\partial^{2} T_{\mathrm{L}}}{\partial Z^{2}}\right), \\
X(Z, V) \leqslant R<\infty,|Z|<\infty, V>0, \\
\left.T_{\mathrm{L}}(R, Z, V)\right|_{V=0}=f_{\mathrm{L}}(R, Z), \quad 1 \leqslant R<\infty,|Z|<\infty, \\
\left.\frac{\partial T_{\mathrm{L}}}{\partial R}\right|_{R=1}=Q_{\mathrm{L}}(Z, V), \quad|Z| \geqslant B(V) .
\end{gathered}
$$

For solid regions

$$
\begin{gathered}
2 \frac{\partial T_{\mathrm{S}}}{\partial V}=V\left(\frac{\partial^{2} T_{\mathrm{S}}}{\partial R^{2}}+\frac{1}{R} \frac{\partial T_{\mathrm{S}}}{\partial R}+\frac{\partial^{2} T_{\mathrm{S}}}{\partial Z^{2}}\right), \\
1 \leqslant R \leqslant X(Z, V),|Z| \leqslant B(V), V>0, \\
\left.\frac{\partial T_{\mathrm{S}}}{\partial R}\right|_{R=1}=\beta Q_{\mathrm{L}}(Z, V), \quad|Z| \leqslant B(V) .
\end{gathered}
$$

For solid-liquid interface conditions

$$
\left.T_{\mathrm{L}}(R, Z, V)\right|_{R=X(Z, V)}=1, \quad|Z| \leqslant B(V)
$$




$$
\begin{gathered}
\left.T_{\mathrm{S}}(R, Z, V)\right|_{R=X(Z, V)}=1, \quad|Z| \leqslant B(V), \\
{\left.\left[1+\left(\frac{\partial X}{\partial Z}\right)^{2}\right]\left(\frac{\partial T_{\mathrm{S}}}{\partial R}-\beta \frac{\partial T_{\mathrm{L}}}{\partial R}\right)\right|_{R=X(Z, V)}} \\
=\frac{2 \lambda}{V} \frac{\partial X}{\partial V}, \quad|z| \leqslant B(V), \\
\left.X(Z, V)\right|_{V=0}=1,\left.\quad B(V)\right|_{V=0}=0, \\
\left.X(Z, V)\right|_{|Z|=B(V)}=1 .
\end{gathered}
$$

$T(R, Z, V)$ is the temperature and the subscripts $\mathrm{L}$ and $\mathrm{S}$ stand for the liquid and solid regions, respectively. All the temperatures have been made dimensionless with the help of the temperature $T_{m}$ (which is unique). Equations (2.4) and (2.7) are the usual Fourier heat conduction equations, and in (2.4) it is assumed that there is no natural convection. Equations (2.6) and (2.8) give the prescribed fluxes over the unsolidified portion and solidified portion of the surface $R=1$, respectively. Equations (2.9) and (2.10) are isotherm conditions and (2.11) is the heat balance condition. In Eqs. (2.4)-(2.13), the following dimensionless variables have been used.

$$
\begin{gathered}
Z=z / R_{0}, \quad R=r / R_{0}, \quad V=2\left(k_{\mathrm{S}} t / R_{0}^{2}\right)^{1 / 2}, \\
\alpha^{2}=k_{\mathrm{S}} / k_{\mathrm{L}}, \quad \lambda=l / c_{\mathrm{S}} T_{m}, \quad \beta=K_{\mathrm{L}} / K_{\mathrm{S}}, \\
Q_{\mathrm{L}}(Z, V)=\hat{Q}_{\mathrm{L}}(z, t) \cdot R_{0} / K_{\mathrm{S}} T_{m}, \quad X(Z, V)=s(z, t) / R_{0}, \\
f_{\mathrm{L}}(R, Z)=\hat{f}_{\mathrm{L}}(r, z) / T_{m}, \quad B(V)=b(t) / R_{0} ;
\end{gathered}
$$

$R_{0}$ is the radius of the cylinder, $K$ is the thermal conductivity, $k$ is the diffusivity, $l$ is the latent heat, and $C_{\mathrm{S}}$ is the specific heat of the solid. Thermal properties are taken to be constants. $Q_{\mathrm{L}}(Z, V)$ is symmetric in $Z$.

The function $f_{\mathrm{L}}(R, Z)$ in Eq. (2.5) is essentially presolidification temperature of the melt, and it can be either prescribed directly or obtained from the solution of a heat conduction problem without phase change with (say) flux prescribed boundary condition. We shall avoid the formulation of this pure heat conduction problem as it is simple; we simply mention here that if $f_{\mathrm{L}}(R, Z)$ is not prescribed it will be the temperature of the melt at time $t=t_{m}, t_{m}>0$, and $t_{m}$ may be obtained, in some cases, using the condition (2.1). The dimensionless time $V$ in Eq. (2.14) in this case can be redefined as

$$
V=2\left\{k_{\mathrm{S}}\left(t-t_{m}\right) / R_{0}^{2}\right\}^{1 / 2}
$$

We shall see later that the short time solution of this pure heat condition problem can also be obtained by the present method.

3. Solution. In view of the condition (2.6), the solution of Eq. (2.4) may be written as

$$
\begin{aligned}
T_{\mathrm{L}}(R, Z, V) & =T_{\mathrm{L}}^{(1)}, & & 1 \leqslant R<\infty,|Z| \geqslant B(V), \\
& =T_{\mathrm{L}}^{(2)}, & & X(Z, V) \leqslant R<\infty,|Z| \leqslant B(V),
\end{aligned}
$$




$$
\begin{gathered}
T_{\mathrm{L}}^{(1)}=\frac{2 \alpha^{3}}{\pi^{1 / 2} V^{3}}\left[\int_{-\infty}^{\infty} \int_{1}^{\infty} H_{1}(p, q) f_{\mathrm{L}}(p, q) d p d q\right. \\
\left.+\int_{-\infty}^{\infty} \int_{0}^{1} H_{1}(p, q) \bar{g}_{\mathrm{L}}(p, q) d p d q\right], \\
T_{\mathrm{L}}^{(2)=} T_{\mathrm{L}}^{(1)}+\frac{2 \alpha^{3}}{\pi^{1 / 2} V^{3}} \int_{-\infty}^{\infty} \int_{0}^{1} H_{1}(p, q) \bar{h}_{\mathrm{L}}(p, q) d p d q, \\
H_{1}(p, q)=p \exp \left[-\alpha^{2}\left\{p^{2}+R^{2}+(Z-q)^{2}\right\} / V^{2}\right] I_{0}\left(2 \alpha^{2} R p / V^{2}\right) .
\end{gathered}
$$

$I_{0}(x)$ is the modified Bessel function of the first kind of order zero, $f_{\mathrm{L}}(R, Z)$ is the known initial temperature, and $\bar{g}_{\mathrm{L}}(R, Z)$ is the unknown initial temperature in the fictitious extension $0 \leqslant R \leqslant 1,|Z|<\infty$, of the original melt region. $\bar{h}_{\mathrm{L}}(R, Z)$ is an unknown function introduced for mathematical convenience.

We shall see later that $T_{\mathrm{L}}^{(1)}$ can be determined by using the condition (2.6) alone and this implies that the temperature in the region $|Z|>B(V)$ is not affected by the growth of the solid layer. For short time, the above assumption is reasonably justified and numerical work has been done in this paper (refer to Fig. 3) to show that this assumption gives insignificant error in the calculation of $T_{\mathrm{L}}^{(1)}$ which is confined to a very small neighbourhood of the points $R=1,|Z|=B(V)$.

The solution of Eq. (2.7) may be written as

$$
\begin{array}{r}
T_{\mathrm{S}}(R, Z, V)=\frac{2}{\pi^{1 / 2} V^{3}}\left[\int_{-\infty}^{\infty} \int_{1}^{\infty} H_{2}(p, q) f_{\mathrm{S}}(p, q) d p d q\right. \\
\left.+\int_{-\infty}^{\infty} \int_{0}^{1} H_{2}(p, q) \bar{g}_{\mathrm{S}}(p, q) d p d q\right], \\
H_{2}(p, q)=p \exp \left[-\left\{p^{2}+R^{2}+(Z-q)^{2}\right\} / V^{2}\right] I_{0}\left(2 R p / V^{2}\right) .
\end{array}
$$

$f_{\mathrm{S}}$ and $\bar{g}_{\mathrm{S}}$ are unknown initial temperatures in the solid regions $1 \leqslant R<\infty,|Z|<\infty$, and $0 \leqslant R \leqslant 1,|Z|<\infty$, respectively. It may be noted that at $V=0$, there is no solid region and therefore these initial temperatures are fictitious. Mathematically, there are six unknowns, namely, $\bar{g}_{\mathrm{L}}, \bar{h}_{\mathrm{L}}, f_{\mathrm{S}}, \bar{g}_{\mathrm{S}}, X(Z, V)$, and $B(V)$ and six conditions (2.6), (2.8)-(2.11), and (2.13) to be satisfied.

For large values of the argument, the following asymptotic expansion for $I_{0}(x)$ can be used [17].

$$
I_{0}(x)=\frac{\exp (x)}{(2 \pi x)^{1 / 2}}\left\{1+\frac{1}{8 x}+\frac{9}{128 x^{2}}+\cdots\right\} .
$$

In Eqs. (3.2), (3.3), and (3.5), $I_{0}(x)$ is replaced by the first two terms of the series in (3.7) and then $T_{\mathrm{L}}$ and $T_{\mathrm{S}}$ are substituted in Eqs. (2.6) and Eqs. (2.8)-(2.11) which, after suitable substitutions, become

$$
\begin{array}{r}
-\pi V Q_{\mathrm{L}}(Z, V)=\int_{\infty}^{-\infty} \int_{0}^{-\infty} D_{1}(V, p) f_{\mathrm{L}}(1-V p / \alpha, Z-V q / \alpha) \exp \left(-p^{2}-q^{2}\right) d p d q \\
+\int_{\infty}^{-\infty} \int_{\alpha / V}^{0}\left\{D_{1}(V, p) /(1-V p / \alpha)^{1 / 2}\right\} \cdot g_{\mathrm{L}}(1-V p / \alpha, Z-V q / \alpha) \\
\cdot \exp \left(-p^{2}-q^{2}\right) d p d q, \quad|Z|<\infty, \quad \text { (3.8) }
\end{array}
$$


$-\pi \beta V Q_{\mathrm{L}}(Z, V)=\int_{\infty}^{-\infty} \int_{0}^{-\infty} D_{2}(V, p) f_{\mathrm{S}}(1-V p, Z-V q) \cdot \exp \left(-p^{2}-q^{2}\right) d p d q$

$$
\begin{array}{r}
+\int_{\infty}^{-\infty} \int_{1 / V}^{0}\left\{D_{2}(V, p) /(1-V p)^{1 / 2}\right\} g_{\mathrm{S}}(1-V p, Z-V q) \\
\cdot \exp \left(-p^{2}-q^{2}\right) d p d q, \quad|Z|<\infty, \quad \text { (3.9) }
\end{array}
$$

$$
\begin{aligned}
\pi X^{3 / 2}= & \int_{\infty}^{-\infty} \int_{X \alpha / V}^{(X-1) \alpha / V} D_{3}(V, p) f_{\mathrm{L}}(X-V p / \alpha, Z-V q / \alpha) \exp \left(-p^{2}-q^{2}\right) d p d q \\
& +\int_{\infty}^{-\infty} \int_{(X-1) \alpha / V}^{-\infty}\left\{D_{3}(V, p) /(X-V p / \alpha)^{1 / 2}\right\}\left\{g_{\mathrm{L}}(X-V p / \alpha, Z-V q / \alpha)\right. \\
& \left.+h_{\mathrm{L}}(X-v p / \alpha, Z-v q / \alpha)\right\} \exp \left(-p^{2}-q^{2}\right) d p d q, \quad|Z| \leqslant B(V), \quad(3.10) \\
\pi X^{3 / 2}= & \int_{\infty}^{-\infty} \int_{X / V}^{(X-1) / V} D_{4}(V, p) f_{\mathrm{S}}(X-V p, Z-V q) \exp \left(-p^{2}-q^{2}\right) d p d q \\
& +\int_{\infty}^{-\infty} \int_{(X-1) / V}^{-\infty}\left\{D_{4}(V, p) /(X-V p)^{1 / 2}\right\} g_{\mathrm{S}}(X-V p, Z-V q)
\end{aligned}
$$

$$
\cdot \exp \left(-p^{2}-q^{2}\right) d p d q, \quad|Z| \leqslant B(V)
$$

$2 \pi \lambda X^{5 / 2} \frac{\partial X}{\partial V}$

$$
\begin{aligned}
= & \left\{1+\left(\frac{\partial X}{\partial Z}\right)^{2}\right\}\left[\beta \int_{\infty}^{-\infty} \int_{X \alpha / V}^{(X-1) \alpha / V} D_{5}(V, p)\right. \\
& \cdot f_{\mathrm{L}}(X-V p / \alpha, z-V q / \alpha) \exp \left(-p^{2}-q^{2}\right) d p d q \\
& +\beta \int_{\infty}^{-\infty} \int_{(X-1) \alpha / V}^{-\infty}\left\{D_{5}(V, p) /(X-V p / \alpha)^{1 / 2}\right\}
\end{aligned}
$$$$
\cdot\left\{g_{\mathrm{L}}(X-V p / \alpha, Z-V q / \alpha)+h_{L}(X-V p / \alpha, Z-V q / \alpha)\right\} \exp \left(-p^{2}-q^{2}\right) d p d q
$$$$
-\int_{\infty}^{-\infty} \int_{X / V}^{(X-1) / V} D_{6}(V, p) f_{\mathrm{S}}(X-V p, Z-V q) \exp \left(-p^{2}-q^{2}\right) d p d q
$$$$
-\int_{\infty}^{\infty} \int_{(X-1) / V}^{\infty}\left\{D_{6}(V, p) /(X-V p)^{1 / 2}\right\} g_{\mathrm{S}}(X-V p, Z-V q)
$$

$$
\begin{aligned}
&\left.\quad \cdot \exp \left(-p^{2}-q^{2}\right) d p d q\right], \quad|Z| \leqslant B(V), \\
& D_{1}(V, p)=2 p \alpha\left(1-V p / \alpha+V^{2} / 16 \alpha^{2}\right)+V(1-V p / \alpha) / 2+3 V^{3} / 32 \alpha^{3}, \\
& D_{2}(V, p)=\left.D_{1}(V, p)\right|_{\alpha=1}, \\
& D_{3}(V, p)=X(X-V p / \alpha)+V^{2} / 16 \alpha^{2}, \\
& D_{4}(V, p)=\left.D_{3}(V, p)\right|_{\alpha=1}, \\
& D_{5}(V, p)=2 p \alpha\left\{X^{2}(X-V p / \alpha)+V^{2} X / 16 \alpha^{2}\right\}+V X(X-V p / \alpha) / 2+3 V^{3} / 32 \alpha^{3}, \\
& D_{6}(V, p)=\left.D_{5}(V, p)\right|_{\alpha=1} \cdot \\
& g_{i}(R, Z)=\bar{g}_{i}(R, Z) / R^{1 / 2}, \quad i=\mathrm{L}, \mathrm{S} ; \\
& h_{\mathrm{L}}(R, Z)=\bar{h}_{\mathrm{L}}(R, Z) / R^{1 / 2} .
\end{aligned}
$$


In writing Eqs. (3.8) and (3.9), the boundary conditions (2.6) and (2.8) have been extended to $|Z|<\infty$ and this is justified as they still satisfy (2.6) and (2.8).

We shall first formally obtain the solution and then justify rigorously some of the steps in the method of solution. Some analytical and numerical checks will also be provided later.

The following series expansions will be assumed for the various known and unknown functions:

$$
\begin{aligned}
& f_{i}(R, Z)=\left.\sum_{n=0}^{\infty} \sum_{m=0}^{\infty} \frac{\partial^{m+n}\left(f_{i}\right)}{\partial R^{m} \partial Z^{n}}\right|_{R=1, Z=0} \frac{(R-1)^{m} Z^{n}}{m ! n !}, \\
& i=\mathrm{L}, \mathrm{S}, 1 \leqslant R<\infty,|Z|<\infty, \\
& g_{i}(R, Z)=\left.\sum_{n=0}^{\infty} \sum_{m=0}^{\infty} \frac{\partial^{m+n}\left(g_{i}\right)}{\partial R^{m} \partial Z^{n}}\right|_{R=1, Z=0} \frac{(R-1)^{m} Z^{n}}{m ! n !} \text {, } \\
& i=\mathrm{L}, \mathrm{S}, 0 \leqslant R \leqslant 1,|Z|<\infty, \\
& Q_{\mathrm{L}}(Z, V)=\sum_{n=1}^{\infty} Q_{\mathrm{L}}^{n}(z) V^{n-1} ; \quad V \geqslant 0,|Z|<\infty, \\
& X(Z, V)=1+\sum_{n=1}^{\infty} A_{n}(Z) V^{n}, \quad V \geqslant 0,|Z| \leqslant B(V), \\
& B(V)=\sum_{n=1}^{\infty} b_{n} V^{n / 2} .
\end{aligned}
$$

The series expansion in (3.16) also holds for $h_{\mathrm{L}}(R, Z)$ (with $g_{i}$ replaced by $h_{\mathrm{L}}$ ). It will be assumed that the series expansions in (3.15)-(3.17) are uniformly convergent in the regions indicated there, and the series expansions in (3.18) and (3.19) are uniformly convergent in some regions of the form $|Z|<Z_{0}, Z_{0}>0 ; 0 \leqslant V \leqslant V_{1}, V_{1}>0$. These assumptions will be further discussed later.

In order to obtain the solution, the series expansions given in (3.15)-(3.18) are substituted in (3.8)-(3.12) and then the limits $V \rightarrow 0+$ of these equations are taken. Five equations in five unknowns are obtained which when solved give a unique solution. Equations (3.8)-(3.12) are then differentiated once with respect to $V$ and limits $V \rightarrow 0+$ are taken. Again five equations in five unknowns are obtained which when solved give a unique solution. This process of higher-order differentiations and limits $V \rightarrow 0+$ can be continued until the desired coefficient in the series (3.18) of the moving boundary is obtained. However, after few differentiations the algebra becomes very complicated. The outcome of the zeroth-order differentiation is given below. $A_{1}(Z)$ is the root of the transcendental Eq. (3.20).

$$
\begin{aligned}
\pi^{1 / 2} \lambda A_{1} \exp \left(A_{1}^{2} \alpha^{2}\right)\left(1-\operatorname{erf} A_{1} \alpha\right)=\beta \alpha\left\{1-f_{\mathrm{L}}(1, Z)\right\}, \quad|Z| \leqslant B(V) . \\
f_{\mathrm{L}}(1, Z)=g_{\mathrm{L}}(1, Z) . \\
f_{\mathrm{S}}(1, Z)=1, \quad g_{\mathrm{S}}(1, Z)=1 . \\
h_{\mathrm{L}}(1, Z)=2\left\{1-f_{\mathrm{L}}(1, Z)\right\} / \operatorname{erfc} A_{1} \alpha .
\end{aligned}
$$


$A_{2}(Z), A_{3}(Z)$, and other related quantities are given in the Appendix. In principle, other coefficients $A_{4}(Z), A_{5}(Z)$, etc., can also be determined, but the algebra becomes extremely complicated. Equations (3.20)-(3.23) need some discussion. It can be easily checked from Eq. (3.20) that $A_{1}(0)=0$ and for $|Z|>0, A_{1}(Z)<0$. It is this property of $A_{1}(Z)$ which enables us to determine $B(V)$. Equation (3.21), together with $h_{\mathrm{L}}(1,0)=0$ which follows from (3.23), implies that at $V=0$, temperature $T_{\mathrm{S}}$ is continuous at $R=1$ and is unity, which it should be.

It may be remarked here that the above method of finding limit $V \rightarrow 0+$ holds even if the temperature solutions given in (3.2), (3.3), and (3.5) are directly substituted in Eqs. (2.6) and (2.8)-(2.11), but in order to find the limit $V \rightarrow 0$, one has to necessarily use the asymptotic expansion of $I_{0}(x)$ given in (3.7). Therefore Eqs. (3.8)-(3.12) are given for the sake of clarity. If the functions $g_{i}(R, Z), i=\mathrm{L}, \mathrm{S}$ are expressed as in Eq. (3.14) then the integrands in (3.8)-(3.12) have no singularities. If more than two terms of the asymptotic expansion in (3.7) are to be used (this asymptotic series does not converge) then Eq. (3.14) can be suitably modified. To determine $B(V)$, condition (2.13) will be used in the following form:

$$
\begin{aligned}
A_{1}^{\prime \prime}(0) & \left(b_{1} V^{1 / 2}+b_{2} V+b_{3} V^{3 / 2}+\cdots\right)^{2} V / 2 \\
& +A_{1}^{\prime \prime \prime \prime}(0)\left(b_{1} V^{1 / 2}+b_{2} V+b_{3} V^{3 / 2}+\cdots\right)^{4} V / 24 \\
& +\left\{A_{2}(0)+\left(b_{1} V^{1 / 2}+b_{2} V+b_{3} V^{3 / 2}+\cdots\right)^{2} A_{2}^{\prime \prime}(0) / 2\right\} V^{2} \\
& +A_{3}(0) V^{3}+\cdots=0, \quad \text { for } V \geqslant 0 .
\end{aligned}
$$

In Eq. (3.24), both $A_{1}(Z)$ and $A_{2}(Z)$ have been expanded in the Maclaurin series. The following two cases arise.

Case I. $A_{2}(0) \neq 0$, i.e., the presolidification dimensionless flux

$$
\left.\beta \frac{\partial f_{\mathrm{L}}}{\partial R}\right|_{R=1, Z=0}
$$

and post-solidification dimensionless flux $\beta Q_{\mathrm{L}}^{(1)}(0)$ are not equal. In this case

$$
\begin{gathered}
b_{1}^{2}=-2 A_{2}(0) / A_{1}^{\prime \prime}(0), \quad A_{1}^{\prime \prime}(0) \neq 0 ; \quad b_{2}=0, \\
b_{3}=-\left\{24 A_{3}(0)+12 b_{1}^{2} A_{2}^{\prime \prime}(0)+b_{1}^{4} A_{1}^{\prime \prime \prime \prime}(0)\right\} /\left(24 b_{1} A_{1}^{\prime \prime}(0)\right), \\
A_{2}(0)=\frac{\beta}{4 \lambda}\left\{Q_{\mathrm{L}}^{(1)}(0)-\left.\frac{\partial f_{\mathrm{L}}}{\partial R}\right|_{R=1, Z=0}\right\}, \\
\pi^{1 / 2} A_{1}^{\prime \prime}(0)=-\left.\frac{\beta \alpha}{\lambda} \frac{\partial^{2} f_{\mathrm{L}}}{\partial Z^{2}}\right|_{R=1, Z=0}, \\
\pi^{1 / 2} A_{1}^{\prime \prime \prime \prime}(0)=\alpha\left\{12\left(A_{1}^{\prime \prime}(0)\right)^{2}-\left.\frac{\beta}{\lambda} \frac{\partial^{4} f_{\mathrm{L}}}{\partial Z^{4}}\right|_{R=1, Z=0}\right\},
\end{gathered}
$$




$$
\begin{gathered}
16 \lambda A_{2}^{\prime \prime}(0)=\left\{4 \frac{\partial^{3} f_{\mathrm{S}}}{\partial R \partial Z^{2}}+4 \pi^{1 / 2} A_{1}^{\prime \prime}(0) \frac{\partial f_{\mathrm{S}}}{\partial R}\right. \\
\left.-2 \beta \frac{\partial^{3}\left(g_{\mathrm{L}}+h_{\mathrm{L}}+f_{\mathrm{L}}\right)}{\partial R \partial Z^{2}}+\beta \frac{\partial^{2}\left(g_{\mathrm{L}}+h_{\mathrm{L}}\right)}{\partial Z^{2}}\right\}\left.\right|_{R=1, Z=0}, \quad, \quad \\
24 \pi^{1 / 2} \lambda A_{3}(0)=21 / 4-2 A_{2}(0)-\beta\left\{21 / 4-2 A_{2}(0) \alpha^{2}\right\} / \alpha \\
+\left[\left(1+4 A_{2}(0)\right) \frac{\partial f_{\mathrm{S}}}{\partial R}+2 \frac{\partial^{2}\left(f_{\mathrm{S}}-g_{\mathrm{S}}\right)}{\partial R^{2}}-\left(3+4 A_{2}(0)\right) \frac{\partial g_{\mathrm{S}}}{\partial R}\right. \\
-\frac{\beta}{\alpha}\left\{-\left(3+4 A_{2}(0) \alpha^{2}\right) \frac{\partial\left(g_{\mathrm{L}}+h_{\mathrm{L}}\right)}{\partial R}+\left(1+4 A_{2}(0) \alpha^{2}\right) \frac{\partial f_{\mathrm{L}}}{\partial R}\right. \\
\left.\left.-2 \frac{\partial^{2}\left(g_{\mathrm{L}}+h_{\mathrm{L}}-f_{\mathrm{L}}\right)}{\partial R^{2}}-\frac{\partial^{2} h_{\mathrm{L}}}{\partial Z^{2}}\right\}\right]\left.\right|_{R=1, Z=0}
\end{gathered}
$$

$X(0, V)=1+A_{2}(0) V^{2}+\cdots$, and since $A_{2}(0)$ is the leading term in the moving boundary, it should be positive. Equation (3.27) confirms this fact. It can be easily shown that $b_{1}$ is real, which it should be, and this also provides a useful check on the method of solution. The solidification spreads along the surface of the cylinder as $O\left(V^{1 / 2}\right), V \ll 1$. $b_{1}$ in Eq. (3.25) will always depend on material parameters. If

$$
\left.\frac{\partial^{2} f_{\mathrm{L}}}{\partial Z^{2}}\right|_{R=1, Z=0}=0 \quad \text { and }\left.\quad \frac{\partial^{4} f_{\mathrm{L}}}{\partial Z^{4}}\right|_{R=1, Z=0} \neq 0
$$

then we modify Eq. (3.19) and write $B(V)=\sum_{n=1}^{\infty} b_{n} V^{n / 4}$. It can be easily shown that the solidification spreads along the surface of the cylinder as $O\left(V^{1 / 4}\right), V \ll 1$.

Case II. $A_{2}(0)=0$. In this case

$$
b_{1}=0 ; \quad b_{2}^{2}=-2 A_{3}(0) / A_{1}^{\prime \prime}(0) .
$$

$X(0, V)=1+A_{3}(0) V^{3}+\cdots$, and since $A_{3}(0)$ is the leading term in the moving boundary it should be positive. To show that $A_{3}(0)$ is actually positive, the mathematical expressions of prescribed initial temperature $f_{\mathrm{L}}(R, Z)$ and prescribed flux $Q_{\mathrm{L}}(R, Z)$ should be known or the numerical values of $A_{3}(0)$ can be obtained. The solidification spreads along the surface of the cylinder as $O(V), V \ll 1$. If

$$
\left.\frac{\partial^{2} f_{\mathrm{L}}}{\partial Z^{2}}\right|_{R=1, Z=0}=0 \quad \text { and }\left.\quad \frac{\partial^{4} f_{\mathrm{L}}}{\partial Z^{4}}\right|_{R=1, Z=0} \neq 0,
$$

then the solidification spreads along the surface as $O\left(V^{1 / 2}\right), V \ll 1 . b_{2}$ depends on the material parameters. It may be mentioned here that even if higher-order terms like $V^{7 / 2}$, $V^{4}$, etc., are considered in Eq. (3.24), the values of the coefficients $b_{1}, b_{2}$, and $b_{3}$ given above in Eqs. (3.25), (3.26), and (3.32) will remain unchanged.

Along with the unknowns in the moving boundary, the unknowns in the temperature solutions given in (3.2), (3.3), and (3.5) are also determined. Only the temperature distribution $T_{\mathrm{L}}^{(2)}(R, Z, V)$ is being given here as $T_{\mathrm{L}}^{(1)}$ and $T_{\mathrm{S}}$ can be easily obtained from 
the expression of $T_{\mathrm{L}}^{(2)}$ given below (after making some minor changes). We replace $I_{0}(x)$ in (3.3) by the first two terms of the asymptotic series in Eq. (3.7), use series expansions for $f_{\mathrm{L}}, \bar{g}_{\mathrm{L}}$, and $\bar{h}_{\mathrm{L}}$ given in (3.15) and (3.16), and integrate term by term. For $V \ll 1$, $X(Z, V) \leqslant R<\infty$, and $|Z| \leqslant B(V), T_{\mathrm{L}}^{(2)}(R, Z, V)$ is given by

$$
\begin{aligned}
T_{\mathrm{L}}^{(2)}(R, Z, V)= & R^{1 / 2}\left\{g_{\mathrm{L}}(1, Z)+\left.(R-1) \frac{\partial g_{\mathrm{L}}}{\partial R}\right|_{R=1}\right\} \operatorname{erfc}\{\alpha(R-1) / V\} / 2 \\
& -\frac{V(R / \pi)^{-1 / 2}}{2 \alpha}\left\{\left.R \frac{\partial g_{\mathrm{L}}}{\partial R}\right|_{R=1}+g_{\mathrm{L}}(1, Z)\right\} \exp \left\{-\alpha^{2}(R-1)^{2} / V^{2}\right\} \\
+ & \frac{1}{2}\left\{f_{\mathrm{L}}(1, Z)+h_{\mathrm{L}}(1, Z)+\left.(R-1) \frac{\partial\left(f_{\mathrm{L}}+h_{\mathrm{L}}\right)}{\partial R}\right|_{R=1}\right\}[1+\operatorname{erfc}(\alpha(R-1) / V)] \\
+ & \frac{\pi^{-1 / 2} V}{4 R \alpha}\left\{f_{\mathrm{L}}(1, Z)+h_{\mathrm{L}}(1, Z)+\left.2 R\left(\frac{\partial f_{\mathrm{L}}}{\partial R}+\frac{\partial h_{\mathrm{L}}}{\partial R}\right)\right|_{R=1} \exp \left\{-\alpha^{2}(R-1)^{2} / V^{2}\right\}\right\} \\
& + \text { terms of the type }(R-1)^{m} V^{n}, \text { where } m+n \geqslant 2, V>0 .
\end{aligned}
$$

Higher-order terms were also calculated in (3.33) and have been included in the numerical work but are not being reported here to save space. In obtaining Eq. (3.33), whenever the limit of integration is $R / V$, it is taken as infinity and this is justified as the integrals are error function integrals [19]. $T_{\mathrm{S}}(R, Z, V)$ can be obtained from Eq. (3.33) by putting $\alpha=1$, replacing the subscript $\mathrm{L}$ by $\mathrm{S}$ everywhere and putting $h_{\mathrm{L}}(R, Z) \equiv 0 . T_{\mathrm{S}}$ is valid only in the region $1 \leqslant R \leqslant X(Z, V),|Z| \leqslant B(V)$. $T_{\mathrm{L}}^{(1)}(R, Z, V)$ can be obtained from Eq. (3.33) by putting $h_{\mathrm{L}}(R, Z) \equiv 0$. $T_{\mathrm{L}}^{(1)}$ can be used only for $|Z|>B(V)$. For the determination of $T_{\mathrm{L}}^{(1)}$, Eq. (3.8) alone is sufficient. It may be noted that in Eq. (3.33), $V$, $|R-1|$, and $|Z|$ can take only small values.

4. Heat conduction without phase change. Short time temperature solution of the pure heat conduction problem with $f_{\mathrm{L}}(R, Z)$ as the prescribed initial temperature of the melt (which need not satisfy Eq. (2.1)) and $Q_{\mathrm{L}}(Z, V)$ as the prescribed flux is given by Eq. (3.8). The unknown $g_{\mathrm{L}}(R, Z)$ and then the temperature can be determined by following the procedure described earlier. Temperature expression is essentially the same as that of $T_{\mathrm{L}}^{(1)}$, which will now be valid for $|Z|<\infty$.

5. Class I problems. Case I. Prescribed flux is finite. If the solidification starts at $V=0$ simultaneously at all points on the surface $R=1$ of the cylinder then the temperature of the melt $f_{\mathrm{L}}(R, Z)$ should be such that

$$
f_{\mathrm{L}}(1, Z)=1, \text { for }|Z|<\infty .
$$

We shall avoid the details of formulation and method of the solution of this class I problem as it requires only some minor changes in the details given earlier and we simply mention here that, in the case of this class I problem,

$$
A_{1}(Z)=0 \quad \text { and } \quad h_{\mathrm{L}}(R, Z) \equiv 0, \quad|Z|<\infty .
$$

$A_{2}(Z)$ and $A_{3}(Z)$ are still given by the Eqs. (B1)-(B10) after deleting Eqs. (B3) and (B8). The solution of this class I problem is not available in the literature. 
Case II. Prescribed flux is infinite. If the melt is superheated and does not satisfy the condition (5.1), then for the solidification to start at $V=0$ it is necessary, and not sufficient, that the prescribed flux is of the form

$$
Q_{\mathrm{L}}(Z, V)=Q_{\mathrm{L}}^{(0)}(Z) / V+Q_{\mathrm{L}}^{(1)}(Z)+Q_{\mathrm{L}}^{(2)}(Z) V+\cdots, \quad V \geqslant 0 .
$$

(Sufficient conditions can be deduced as in [15], Eq. (32).) In this case also the present method of solution is valid. Most of the results given earlier, and those in the Appendix, can be used for this problem also with some appropriate changes.

\section{Justification of some of the steps in the method of solution and comparison with other} works. To save space, only a few important points are being discussed.

(i) The assumptions made earlier about the uniform convergence of the series expansions for unknown functions are a priori and the convergence can be established only if they are determined completely, which is not possible in the present problem. In such a situation, what is done in many problems of mathematical physics and has been done in this paper also, is to check whether the final outcome is all right, and for that several mathematical checks have been given earlier and, further, the numerical results also provide some checks. These assumptions ensure the differentiation of integrals in Eqs. (3.8)-(3.12) (with respect to $V$ and taking the limit $V \rightarrow 0+$ ), justify the term by term integration for determining the temperature, and are sufficient to invoke Watson's Lemma (see below). There does not seem to be any rigorous way to find the region of convergence of the series for the moving boundary except by obtaining the numerical values of the coefficients for a good estimate. It can be shown that derivatives of $A_{1}(Z), A_{2}(Z)$, etc., of all orders exist at $Z=0$, but the validity of their Maclaurin series expansions cannot be established analytically.

(ii) In the first integral of Eq. (3.2), the limits of integration for the variable $p$ from 1 to $\infty$ and for $q$ from $-\infty$ to $+\infty$ are dictated by the region of the known initial temperature $f_{\mathrm{L}}(R, Z)$ of the melt and so these limits of integrations cannot be altered. If the procedure mentioned earlier for determining the unknowns is followed, then the coefficients $A_{1}(Z)$, $Z_{2}(Z)$, etc., in the moving boundary remain unaltered even if the lower limit for $p$ in the second integral of Eq. (3.2) is changed from 0 to $C$, where $C$ could be any real number less than one. Using Watson's Lemma [20] or the properties of error function integrals, it can be shown that for very short time (asymptotic analysis) so long as $C \neq 1, C$ can be chosen as any real number less than one and the different values of $C$ make insignificant changes in the value of the integral. This asymptotic analysis has been indicated mainly to justify rigorously the solution, but in actual practice the solution may be valid for much longer time (see numerical work). Similar types of arguments can be put forward for the possible changes in the limits for the variable $p$ in other integrals and are not being repeated here. It may be remarked here that it is not possible to alter the limits for the variable of integration $q$ (except changing the limits from 0 to $\infty$ because of symmetry) and still obtain the solution, and nowhere has the two-dimensional limit $R \rightarrow 1, V \rightarrow 0+$ of the integrals been taken which in fact does not exist. 
(iii) In this paper, for all practical purposes, $X(Z, V)$ is given by

$$
\begin{aligned}
X(Z, V)= & 1+A_{1}^{\prime \prime}(0) Z^{2} V / 2+A_{11}^{\prime \prime \prime}(0) Z^{4} V / 24 \\
& +A_{2}(0) V^{2}+A_{2}^{\prime \prime}(0) Z^{2} V^{2} / 2+A_{3}(0) V^{3} .
\end{aligned}
$$

Equation (6.1) can be rewritten as

$$
X(Z, V)=1+A_{2}(0) V^{2}\left(1-\frac{Z^{2}}{b_{1}^{2} V}\right)+\cdots .
$$

For $V \ll 1$, the propagation of solidification towards the interior is proportional to $V^{2}$. Similarly, if $A_{2}(0)=0$ it can be shown that the solidification propagation towards the interior is proportional to $V^{3}$. These behaviors of the moving boundary are in conformity with the well-known results given in [4]. The shapes of the moving boundaries in [6-8] and in the present work also appear to be similar.

7. Numerical work. For plotting the moving boundary or temperature distribution, a pertinent question which can be asked is: What is the range of time for which these short

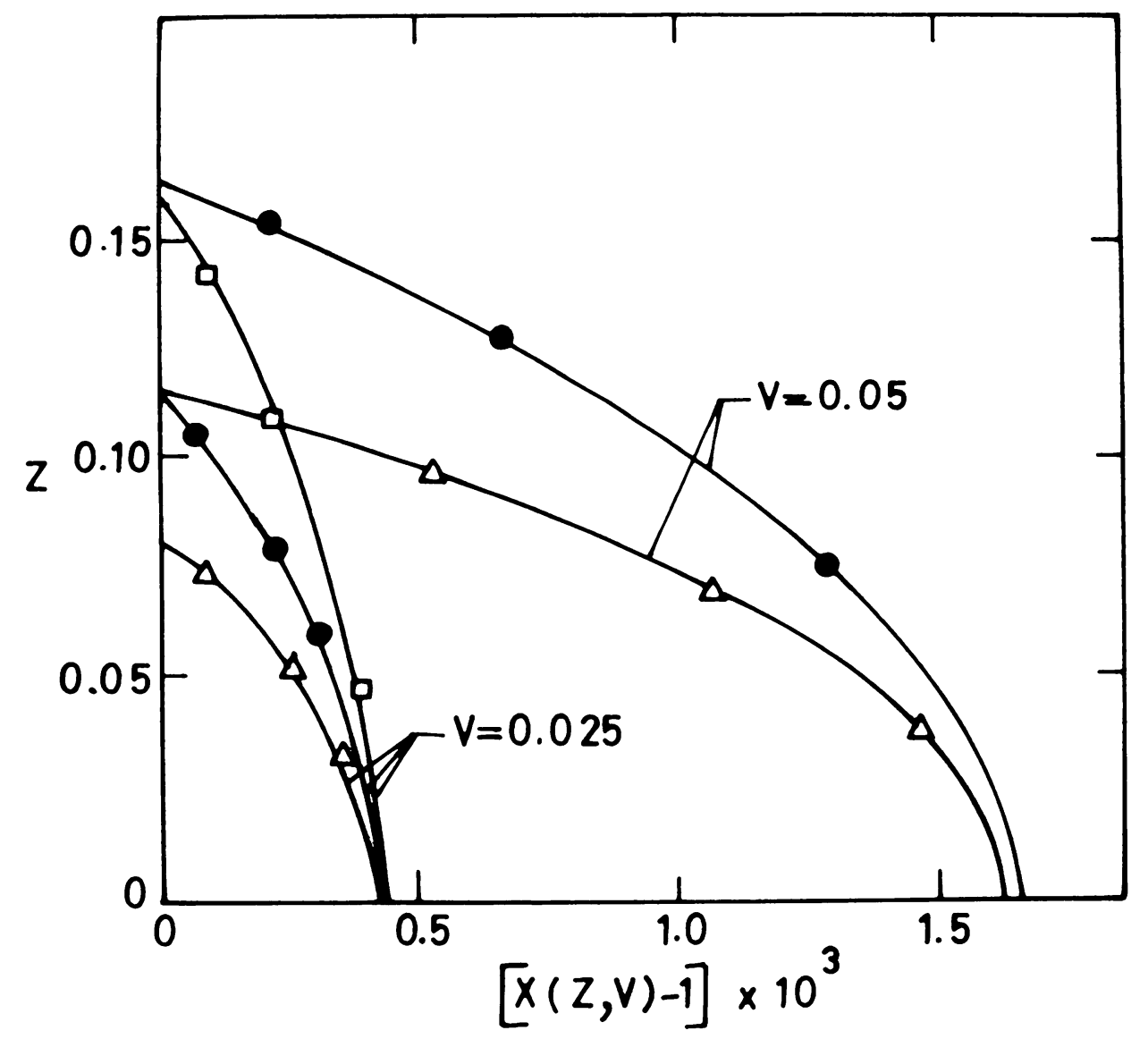

FIG. 1. Effect of varying initial temperature on the solidification front. $\lambda=0.563, \beta=0.429, \alpha=1.538$. $Q_{\mathrm{L}}(Z, V)=2\left\{1+\exp \left(-Z^{2}\right)\right\}$, and $f_{\mathrm{L} .}(R, Z)=1.5-0.5 \exp \left\{-\left(d Z^{2}+R\right)\right\}$, where $d$ takes the values 2,4 , and 8 for the curves $-\square-,-\bullet-$, and $-\triangle-$, respectively. 
time solutions are valid? The answer is simple. We consider the series for $B(V)$ in Eq. (3.19). If the absolute values of the coefficients $b_{1}, b_{2}, b_{3}$, etc., go on decreasing (for a given set of parameter values), then by calculating $\left|b_{n} V^{n / 2}\right|$ for a given $V$ (where $b_{n}$ is the last coefficient calculated in $B(V))$ it can be found out whether $\left|b_{n+1} V^{(n+1) / 2}\right|$ will contribute significantly toward the calculation of $\sum_{m=1}^{n} b_{m} V^{m / 2}$ (it is assumed here that the absolute values of the coefficients $b_{n+1}, b_{n+2}$, etc., will also go on decreasing) and this will determine the range of time for which the solution is valid. If only one coefficient is available, then $V$ can be taken arbitrarily to be very small. For one-dimensional class I problems studied in [21], the validity of this type of criterion for the series $\sum_{m=1}^{n} A_{m} V^{m}$ has already been verified with the help of numerical schemes, but since $A_{1}$ is of a different nature in the present work, we had to evolve the criterion in terms of the coefficients $b_{1}$, $b_{2}$, etc. The numerical results presented in Figs. 1-3 justify this criterion.

In Fig. 1, flux is kept constant and the initial temperature is varied. For short time an increase in the initial temperature decreases the spread of solidification along the surface much faster than the solidification thickness. In Fig. 2, initial temperature is kept constant

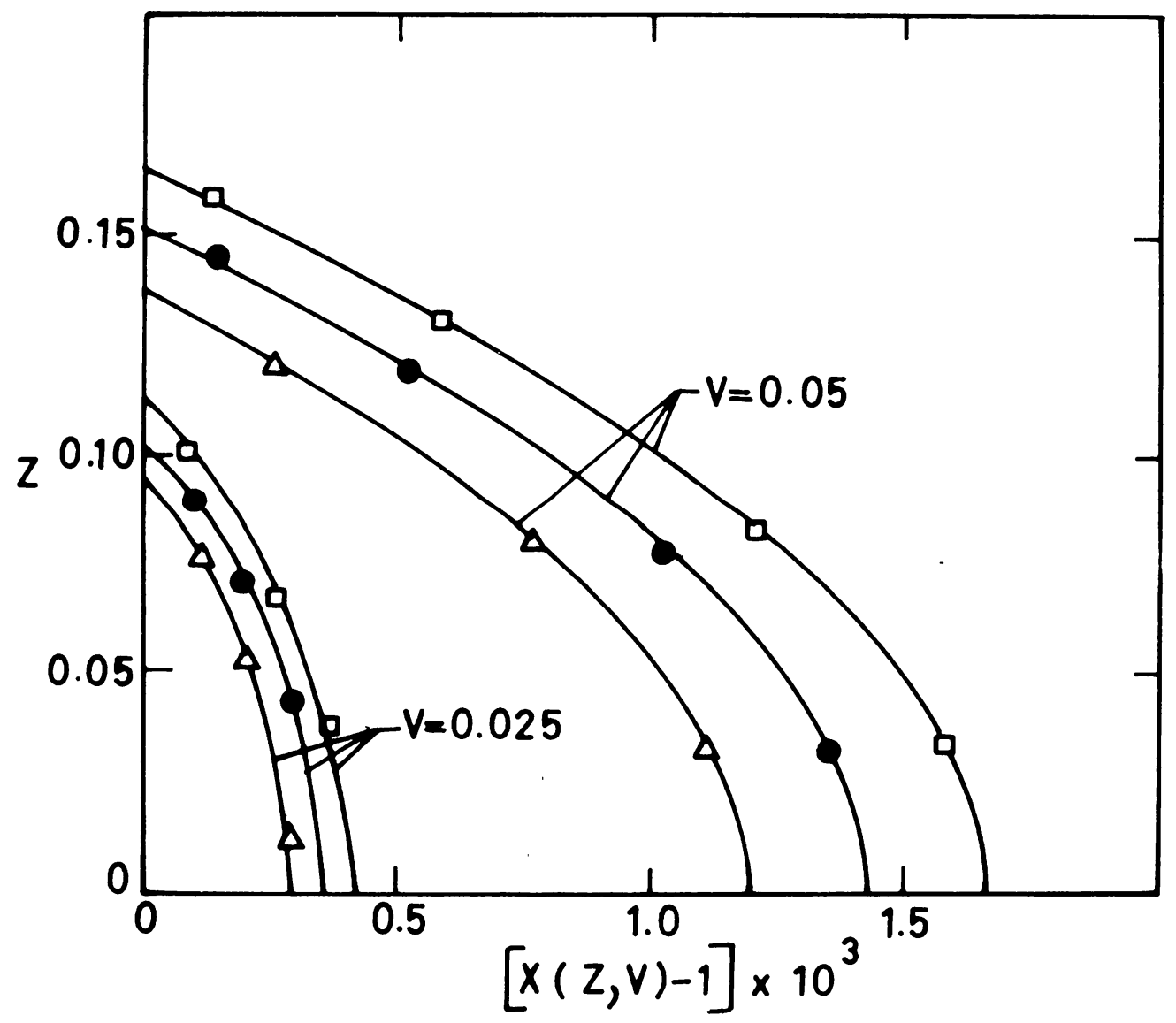

FIG. 2. Effect of varying flux on the solidification front. $f_{\mathrm{L}}(R, Z)=1.5-0.5 \exp \left\{-\left(4 Z^{2}+R\right)\right\}$ and $Q_{\mathrm{L}}(Z, V)$ $=2\left\{m+\exp \left(-Z^{2}\right)\right\}$ where $m$ takes the values $1,0.75$, and 0.5 for the curves $-\square-,-\bullet_{-}$, and $-\triangle-$, respectively. 


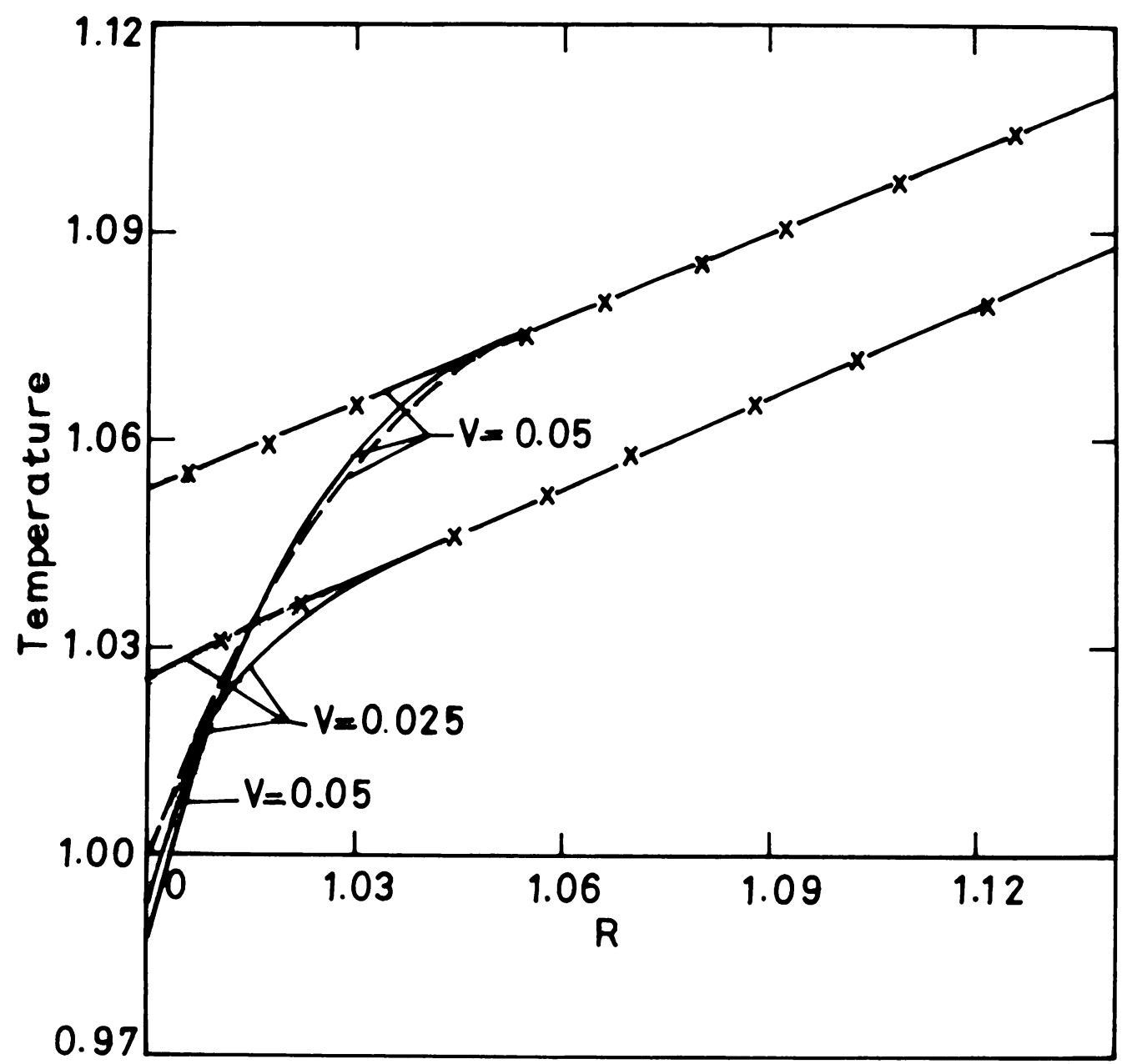

FIG. 3. Comparison of initial temperature $f_{\mathrm{L}}(R, Z)$, temperatures $T_{\mathrm{L}}^{(1)}$ and $T_{\mathrm{L}}^{(2)}$ (Eqs. (3.2) and (3.3)) at $Z=B(V)$ and different values of $R . Q_{\mathrm{L}}(Z, V)=2\left\{1+\exp \left(-Z^{2}\right)\right\}$ and $f_{\mathrm{L}}(R, Z)=1.5-0.5 \exp \left\{-\left(8 Z^{2}+\right.\right.$ $R)\}$. For $V=0.025$ and $0.05, B(V)=0.0806$ and 0.1162 , respectively. The curves $-\times-,-,---$ are for $f_{\mathrm{L}}$, $T_{\mathrm{L}}^{(1)}$, and $T_{\mathrm{L}}^{(2)}$, respectively.

and flux is varied. An increase in the prescribed flux increases the solidification along the interior more rapidly than along the surface. The maximum values of the ratios $B(V) /|1-X(0, V)|$ in Figs. 1 and 2 are 386.2 and 274.5, respectively. In all the figures the case $A_{2}(0) \neq 0$ is considered. $b_{1}=1.0040,0.7991,0.5020$ and $b_{3}=0.4891,0.4233$, 0.3004 for the three initial temperatures in the same order as mentioned in Fig. 1. $b_{1}=1.0799,0.6573,0.6000$ and $b_{3}=0.4233,0.3351,0.2549$ for the three different fluxes in the same order as mentioned in Fig. 2.

It is apparent that the effect of temperature $T_{\mathrm{L}}^{(2)}$ on $T_{\mathrm{L}}^{(1)}$ (given by Eqs. (3.2) and (3.3)) for a given $V$ will be maximum at $Z=B(V), R \geqslant 1$. In Fig. 3, the absolute difference between $T_{\mathrm{L}}^{(2)}$ and $T_{\mathrm{L}}^{(1)}$ is maximum at $Z=B(V), R=1$ (which it should be) and is about 
$1.7 \%$. This difference is decreasing very fast as $R$ increases. The effect of solidification on $T_{\mathrm{L}}^{(1)}$ is felt mainly in a very small neighborhood of the point $Z=B(V), R=1$ and the assumption that $T_{\mathrm{L}}^{(2)}$ does not affect $T_{\mathrm{L}}^{(1)}$ appreciably is justified.

Aluminum melt is considered for numerical work. Since the solidified thickness is very small it does not seem to be interesting to calculate the temperature in the solid.

8. Concluding remarks. The method of solution presented in this paper is quite simple as compared to the complexity of the problem considered. With suitable modifications the method is applicable to some other geometries and some other types of boundary conditions also. The validity of the short time analytical solution has been reasonably established. The results given in Eqs. (3.25), (3.26), and (3.32) are new in the literature. A more general problem could have been to consider the interactions of several cold spots on the surface of the cylinder, but at present there does not seem to be any systematic way to tackle this general problem. It is hoped that the present work will motivate further research work in this direction.

Appendix. $A_{2}(Z), A_{3}(Z)$, and other related quantities are as follows:

$$
\begin{aligned}
& 16 \lambda A_{2}(Z)\left\{1+A_{1}^{2} \alpha^{2}\right\} \\
& =\left[-20 \lambda A_{1}^{2}+\left.2 \frac{\partial g_{\mathrm{S}}}{\partial R}\right|_{R=1} \operatorname{erfc} A_{1}+\operatorname{erfc} A_{1}+\left.2\left(1+\operatorname{erf} A_{1}\right) \frac{\partial f_{\mathrm{S}}}{\partial R}\right|_{R=1}\right. \\
& \quad-\beta\left\{\left.2 \frac{\partial\left(g_{\mathrm{L}}+h_{\mathrm{L}}\right)}{\partial R}\right|_{R=1} \operatorname{erfc} A_{1} \alpha+g_{\mathrm{L}}(1, Z) \operatorname{erfc} A_{1} \alpha+\left.2\left(1+\operatorname{erf} A_{1} \alpha\right) \frac{\partial f_{\mathrm{L}}}{\partial R}\right|_{R=1}\right. \\
& \left.\left.+\pi^{-1 / 2}\left(8 A_{1} \alpha \exp \left(-A_{1}^{2} \alpha^{2}\right)-\operatorname{erfc}\left(A_{1} \alpha\right)\right) h_{\mathrm{L}}(1, Z)\right\}\right] . \quad \text { (B1) }
\end{aligned}
$$

$\left.2 i \operatorname{erfc}\left(A_{1} \alpha\right) \frac{\partial\left(g_{\mathrm{L}}+h_{\mathrm{L}}\right)}{\partial R}\right|_{R=1}$

$$
\begin{gathered}
=A_{1} \alpha\left(7-\operatorname{erf} A_{1} \alpha\right) f_{\mathrm{L}}(1, Z)+4 A_{1} \alpha \operatorname{erfc}\left(A_{1} \alpha\right) h_{\mathrm{L}}(1, Z) \\
-6 A_{1} \alpha-\pi^{-1 / 2} \exp \left(-A_{1}^{2} \alpha^{2}\right) \times\left\{f_{\mathrm{L}}(1, Z)+2 h_{\mathrm{L}}(1, Z)\right\} \\
+\left.2\left(2 A_{1} \alpha+i \operatorname{erfc} A_{1} \alpha\right) \frac{\partial f_{\mathrm{L}}}{\partial R}\right|_{R=1}-4 A_{2} \alpha^{2} \exp \left(-A_{1}^{2} \alpha^{2}\right) h_{\mathrm{L}}(1, Z) \\
\left.\frac{\partial g_{\mathrm{L}}}{\partial R}\right|_{R=1}=2 Q_{L}^{(1)}-\left.\frac{\partial f_{\mathrm{L}}}{\partial R}\right|_{R=1}-f_{L}(1, Z) / 2 . \\
\left.\frac{\partial g_{\mathrm{S}}}{\partial R}\right|_{R=1}=2 \beta Q_{\mathrm{L}}^{(1)}-\left.\frac{\partial f_{\mathrm{S}}}{\partial R}\right|_{R=1}-1 / 2 . \\
\left.2\left(A_{1}+i \operatorname{erfc} A_{1}\right) \frac{\partial g_{\mathrm{S}}}{\partial R}\right|_{R=1}=2\left(2 A_{1}+i \operatorname{erfc} A_{1}\right) \beta Q_{\mathrm{L}}^{(1)}-A_{1}-i \operatorname{erfc} A_{1} .
\end{gathered}
$$




$$
\begin{aligned}
8 \pi^{1 / 2} \lambda & \left(3+2 A_{1}^{2} \alpha^{2}\right) A_{3}(Z) \\
= & -30 \lambda \pi^{1 / 2} A_{1}\left(A_{2}+A_{1}^{2} / 4\right)+8 \lambda \pi^{1 / 2} A_{1} A_{1}^{\prime 2}+2 \pi^{1 / 2} A_{1} \operatorname{erfc} A_{1} \\
& +2\left(21 / 8-A_{2}\right) \exp \left(-A_{1}^{2}\right)+\left.\left\{7 \pi^{1 / 2} A_{1} \operatorname{erfc} A_{1}-\left(3+4 A_{2}\right) \exp \left(-A_{1}^{2}\right)\right\} \frac{\partial g_{\mathrm{S}}}{\partial R}\right|_{R=1} \\
& +\left\{5 \pi^{1 / 2} A_{1}\left(1+\operatorname{erfc} A_{1}\right)+\left(4 A_{2}+1\right) \exp \left(-A_{1}^{2}\right)\right\}-\left.2 \pi^{1 / 2} \operatorname{erfc} A_{1} \frac{\partial^{2} g_{\mathrm{S}}}{\partial R^{2}}\right|_{R=1} \\
& +\left.2\left\{\exp \left(-A_{1}^{2}\right)+\pi^{1 / 2} A_{1}\left(1+\operatorname{erfc} A_{1}\right)\right\} \frac{\partial^{2} f_{\mathrm{S}}}{\partial R^{2}}\right|_{R=1} \\
& -\frac{\beta}{\alpha}\left[2 \left\{\pi^{1 / 2} A_{1} \alpha \operatorname{erfc} A_{1} \alpha+\left(3-6 A_{2} \alpha^{2}-2 A_{1}^{2} \alpha^{2}-4 A_{1}^{2} A_{2}^{2} \alpha^{6}\right.\right.\right. \\
& \left.+2\left\{\pi_{1}^{2} A_{2} \alpha^{4}+2 A^{4} \alpha^{4}+A_{2} \alpha^{2}+4 A_{1} A_{3} \alpha^{4}\right) \exp \left(-A_{1}^{2}\right)\right\} h_{\mathrm{L}}(1, Z) \\
& +\left\{7 \pi^{1 / 2} A_{1} \alpha-\left(3+4 A_{2} \alpha+\left(21 / 8-A_{2} \alpha^{2}\right) \exp \left(-A_{1}^{2} \alpha^{2}\right)\right\} f_{\mathrm{L}}(1, Z)\right. \\
& \left.\left.+\left.2\left\{\left(1+4 A_{2} \alpha^{2}\right) \exp \left(-A_{1}^{2} \alpha^{2}\right)+5 \pi^{1 / 2} A_{1} \alpha\left(1+\operatorname{erfc} A_{1} \alpha\right)\right\} \cdot \frac{\partial f_{\mathrm{L}}}{\partial R}\right|_{R=1} A_{1}^{\prime}(Z)=\frac{d A_{1}}{d Z} \cdot A_{1}^{2} \alpha^{2}\right)\right\}\left.\frac{\partial\left(g_{\mathrm{L}}+h_{\mathrm{L}}\right)}{\partial R}\right|_{R=1} \\
& -\left.2 \pi^{1 / 2} i \operatorname{erfc} A_{1} \alpha \frac{\partial^{2}\left(g_{\mathrm{L}}+h_{\mathrm{L}}\right)}{\partial R^{2}}\right|_{R=1} \\
& +\left.2\left\{\pi^{1 / 2} A_{1} \alpha\left(1+\operatorname{erfc} A_{1} \alpha\right)+\exp \left(-A_{1}^{2} \alpha^{2}\right)\right\} \frac{\partial^{2} f_{\mathrm{L}}}{\partial R^{2}}\right|_{R=1} \\
& \left.-\left.\exp \left(-A_{1}^{2} \alpha^{2}\right) \frac{\partial^{2} h_{\mathrm{L}}}{\partial Z^{2}}\right|_{R=1}\right], \\
& \\
&
\end{aligned}
$$

$$
\begin{aligned}
4 i^{2} \operatorname{erfc} & \left.\left(A_{1} \alpha\right) \frac{\partial^{2}\left(g_{\mathrm{L}}+h_{\mathrm{L}}\right)}{\partial R^{2}}\right|_{R=1} \\
= & -\left.\left\{2 A_{1} \alpha \exp \left(-A_{1}^{2} \alpha^{2}\right) \pi^{-1 / 2}+\left(1+2 A_{1}^{2} \alpha^{2}\right)\left(1+\operatorname{erf} A_{1} \alpha\right)\right\} \frac{\partial^{2} f_{\mathrm{L}}}{\partial R^{2}}\right|_{R=1} \\
& +\left.2\left\{4 A_{1} \alpha i \operatorname{erfc} A_{1} \alpha-\left(1+2 A_{2} \alpha^{2}\right) \operatorname{erfc} A_{1}\right\} \frac{\partial\left(g_{\mathrm{L}}+h_{\mathrm{L}}\right)}{\partial R}\right|_{R=1} \\
& -\left.\operatorname{erfc} A_{1} \alpha \frac{\partial^{2} h_{\mathrm{L}}}{\partial Z^{2}}\right|_{R=1}-\left.2 \frac{\partial^{2} f_{\mathrm{L}}}{\partial Z^{2}}\right|_{R=1} \\
& -\left\{\operatorname{erfc} A_{1} \alpha-10 A_{1} \alpha i \operatorname{erfc} A_{1} \alpha\right.
\end{aligned}
$$




$$
\begin{aligned}
& \left.+8 A_{2} \alpha^{2} \operatorname{erfc} A_{1} \alpha+48 A_{2} \alpha^{2}+12 A_{1}^{2} \alpha^{2}\right\} f_{\mathrm{L}}(1, Z) / 4+48 A_{2} \alpha^{2} \\
& +12 A_{1}^{2} \alpha^{2}-\left\{6 \pi^{-1 / 2} A_{1} \alpha \exp \left(-A_{1}^{2} \alpha^{2}\right)\right. \\
& \left.+\left(1+4 A_{2} \alpha^{2}+6 A_{1}^{2} \alpha^{2}\right)\left(1+\operatorname{erf} A_{1} \alpha\right)\right\}\left.\frac{\partial f_{\mathrm{L}}}{\partial R}\right|_{R=1} \\
& +4\left\{-\operatorname{erfc}\left(A_{1} \alpha\right)\left(A_{1}^{2} \alpha^{2}+2 A_{2} \alpha^{2}+1 / 16\right)+\pi^{-1 / 2} A_{1} \alpha\right. \\
& \left.\cdot\left(1+2 A_{2} \alpha^{2}-2 A_{2}^{2} \alpha^{4}\right) \exp \left(-A_{1}^{2} \alpha^{2}\right)\right\} h_{\mathrm{L}}(1, Z) \\
& -16 \lambda A_{1} \alpha^{2} A_{3} / \beta \text {. } \\
& \left.2 \frac{\partial^{2} g_{\mathrm{L}}}{\partial R^{2}}\right|_{R=1}=\left.2 \frac{\partial^{2} f_{\mathrm{L}}}{\partial R}\right|_{R=1}-4 \pi^{1 / 2} \alpha Q_{L}^{(2)}+\left.\frac{\partial f_{\mathrm{L}}}{\partial R}\right|_{R=1}-\left.3 \frac{\partial g_{\mathrm{L}}}{\partial R}\right|_{R=1} \text {. } \\
& \left.2 \frac{\partial^{2} g_{\mathrm{S}}}{\partial R^{2}}\right|_{R=1}=\left.2 \frac{\partial^{2} f_{\mathrm{S}}}{\partial R^{2}}\right|_{R=1}-4 \pi^{1 / 2} \beta Q_{L}^{(2)}+\left.\frac{\partial f_{\mathrm{S}}}{\partial R}\right|_{R=1}-\left.3 \frac{\partial g_{\mathrm{S}}}{\partial R}\right|_{R=1} . \\
& =\left.\left\{2 A_{1} \exp \left(-A_{1}^{2}\right) \pi^{-1 / 2}+\left(1+2 A_{1}^{2}\right)\left(1+\operatorname{erf} A_{1}\right)\right\} \frac{\partial^{2} f_{\mathrm{S}}}{\partial R^{2}}\right|_{R=1} \\
& -\left.2\left\{4 A_{1} i \operatorname{erfc} A_{1}-\left(1+2 A_{2}\right) \operatorname{erfc} A_{1}\right\} \frac{\partial g_{\mathrm{S}}}{\partial R}\right|_{R=1} \\
& +\left.2 \frac{\partial^{2} f_{\mathrm{S}}}{\partial Z^{2}}\right|_{R=1}+\left.\left\{6 \pi^{-1 / 2} A_{1} \exp \left(-A_{1}^{2}\right)+\left(1+\operatorname{erfc} A_{1}\right)\left(1+6 A_{1}^{2}+4 A_{2}\right)\right\} \frac{\partial f_{\mathrm{S}}}{\partial R}\right|_{R=1} \\
& +\left\{\operatorname{erfc} A_{1}\left(1+8 A_{2}\right)-10 A_{1} i \operatorname{erfc} A_{1}\right\} / 4 \text {. }
\end{aligned}
$$

Acknowledgment. The author wishes to thank Professor P. L. Sachdev, Department of Applied Mathematics and Professor A. K. Lahiri, Department of Metallurgy, Indian Institute of Science, Bangalore, for some useful discussions.

\section{REFERENCES}

[1] J. C. Meuhlbauer and J. E. Sunderland, Heat conduction with freezing or melting, Appl. Mech. Reviews 18, 951-959 (1965)

[2] J. R. Ockendon and W. R. Hodgkins, Moving boundary problems in heat flow and diffusion, Clarendon Press, Oxford, 1975

[3] D. G. Wilson, A. D. Solomon, and P. T. Boggs, eds., Moving boundary problems, Academic Press, 1978

[4] D. L. Sikarskie and B. A. Boley, The solution of a class of two-dimensional melting and solidification problems, Internat. J. Solids and Structures 1, 207-234 (1965)

[5] B. A. Boley, An applied overview of moving boundary problems, in Moving Boundary Problems, edited by D. G. Wilson, A. D. Solomon, and P. T. Boogs, pp. 205-231, Academic Press, 1978

[6] H. P. Yagoda and B. A. Boley, Starting solution of a slab under plane or axisymmetric hot spot, Quart. J. Mech. Appl. Math. 23, 225-246 (1970)

[7] B. A. Boley and H. P. Yagoda, The starting solution for two-dimensional heat conduction problems with change of phase, Quart. Appl. Math. 27, 223-246 (1969) 
[8] B. A. Boley and H. P. Yagoda, The three-dimensional starting solution for a melting slab, Proc. Roy. Soc. London Ser A 323, 89-110 (1971)

[9] M. A. Boles and M. N. Oz̈isik, Exact solution for freezing in cylindrical symmetry, ASME J. Heat Transfer 105, 401-403 (1983)

[10] L. N. Tao, The Stefan problem with arbitrary initial and houndary conditions, Quart. Appl. Math. 36, 223-233 (1978)

[11] R. I. Pedroso and G. A. Damoto, Perturhation solutions for spherical solutions of saturated liquids, ASME J. Heat Transfer 95, 42-46 (1973)

[12] T. R. Goodman, The heat balance integral-further considerations and refinements, ASME J. Heat Transfer 83, 81-85 (1961)

[13] S. C. Gupta, Two-dimensional heat conduction with phase change in a semi-infinite mold, Internat. J. Engrg. Sci. 19, 137-146 (1981)

[14] S. C. Gupta, Two-dimensional solidification in a cylindrical mold with imperfect mold contact, Internat. J. Engrg. Sci. 23, $901-913(1985)$

[15] B. H. Kear, E. M. Breinan, and L. E. Greenwald, Laser glazing-a new process for production and control of rapidly chilled metallurgical micro-structures, Metals Technology 6, 121-129 (1979)

[16] B. A. Boley, A method of heat conduction analysis of melting and solidification problems, J. Math. Phys. 40. 300-313 (1961)

[17] H. S. Carslaw and J. C. Jaeger, Conduction of heat in solids, Clarendon Press, Oxford, 1959

[18] B. A. Boley, Proceedings of the eleventh international congress of applied mechanics, Munich, 586-596, 1964

[19] M. Abramovitz and I. A. Stegun (eds.), Handbook of mathematical functions, Dover, 1972

[20] H. Jeffreys, Asymptotic approximations, Clarendon Press, Oxford, 1962

[21] R. Vellingiri, Solutions of some radially symmetric problems of heat conduction with phase change, Ph.D. Thesis, Indian Institute of Science, Bangalore, 1983 